\title{
Novel GDAP1 Mutation in a Vietnamese Family with Charcot-Marie-Tooth Disease
}

\author{
Phuong-Thao Mai $\mathbb{D},{ }^{1}$ Dong-Truc Le, ${ }^{2}$ Tan-Trung Nguyen $\left(\mathbb{D},{ }^{3}\right.$ Hoang-Linh Le Gia, ${ }^{2}$ \\ Trung-Hieu Nguyen Le, ${ }^{4}$ Minh Le, ${ }^{5}$ and Duc-Minh Do (iD ${ }^{2}$ \\ ${ }^{1}$ Department of Physiology, Faculty of Medicine, University of Medicine and Pharmacy at Ho Chi Minh City, Vietnam \\ ${ }^{2}$ Center for Molecular Biomedicine, University of Medicine and Pharmacy at Ho Chi Minh City, Vietnam \\ ${ }^{3}$ Department of Biotechnology, Faculty of Chemical Engineering, Ho Chi Minh City University of Technology, VNU-HCM, Vietnam \\ ${ }^{4}$ Department of Neurology, Faculty of Medicine, University of Medicine and Pharmacy at Ho Chi Minh City, Vietnam \\ ${ }^{5}$ Department of Neurology, University Medicine Center at Ho Chi Minh City, Vietnam
}

Correspondence should be addressed to Duc-Minh Do; ducminh@ump.edu.vn

Received 15 October 2018; Revised 21 December 2018; Accepted 14 April 2019; Published 24 April 2019

Academic Editor: Przemko Tylzanowski

Copyright ( 2019 Phuong-Thao Mai et al. This is an open access article distributed under the Creative Commons Attribution License, which permits unrestricted use, distribution, and reproduction in any medium, provided the original work is properly cited.

Background. Mutations of GDAP1 gene cause autosomal dominant and autosomal recessive Charcot-Marie-Tooth (CMT) disease and over 80 different mutations have been identified so far. This study analyzed the clinical and genetic characteristics of a Vietnamese CMT family that was affected by a novel GDAP1 mutation. Methods. We present three children of a family with progressive weakness, mild sensory loss, and absent tendon reflexes. Electrodiagnostic analyses displayed an axonal type of neuropathy in affected patients. Sequencing of GDAP1 gene was requested for all members of the family. Results. All affected individuals manifested identical clinical symptoms of motor and sensory impairments within the first three years of life, and nerve conduction study indicated the axonal degeneration. A homozygous GDAP1 variant (c.667_671dup) was found in the three affected children as recessive inheritance pattern. The mutation leads to a premature termination codon that shortens GDAP1 protein (p.Gln224Hisfs*37). Further testing showed heterozygous c.667_671dup variant in the parents. Discussion. Our study expands the mutational spectrum of GDAP1-related CMT disease with the new and unreported GDAP1 variant. Alterations in GDAP1 gene should be evaluated as CMT causing variants in the Vietnamese population, predominantly axonal form of neuropathy in CMT disease.

\section{Introduction}

Charcot-Marie-Tooth disease (CMT) is one of the most common inherited peripheral neuropathies affecting motor and sensory neurons. More than 80 causative genes have been reported as autosomal dominant, autosomal recessive, and Xlinked forms [1-3]. The disease features can be diverse even among those sharing the same mutation. Assessment of age at onset, key clinical findings (the dominance of motor or sensory, upper or lower limbs symptoms, and differentiating features), and family history are very useful in choosing a genetic testing strategy. The classical phenotype is usually characterized by early age at onset with slowly progressive weakness and atrophy of the distal muscles mostly in the lower limbs, foot deformities, walking impairment, areflexia, and mild sensory deficits [4]. Clinically, patients are classified into 3 groups based on the nerve conduction studies: a demyelinating (CMT1) with very slow motor nerve conduction velocity (MNCV), an axonal (CMT2) with normal or slight reduction of $\mathrm{MNCV}$, and an intermediate phenotype. The demyelinating type occurs $2 / 3$ of CMT cases with the duplication in PMP22 gene, while the mutation spectrum in CMT2 is more diverse including variants in MFN2, MPZ, $G J B 1$, and GDAP1 and a large number of additional genes [5].

GDAP1 (Ganglioside-induced differentiation-associatedprotein 1) is an integral membrane protein of the outer mitochondrial membrane with a ubiquitous tissue distribution but predominantly expressed in neurons. It is involved in many 


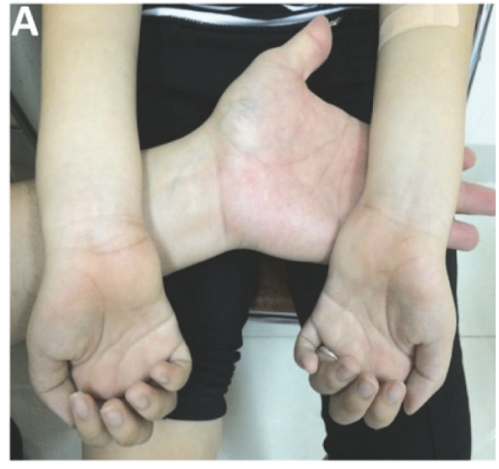

(a)

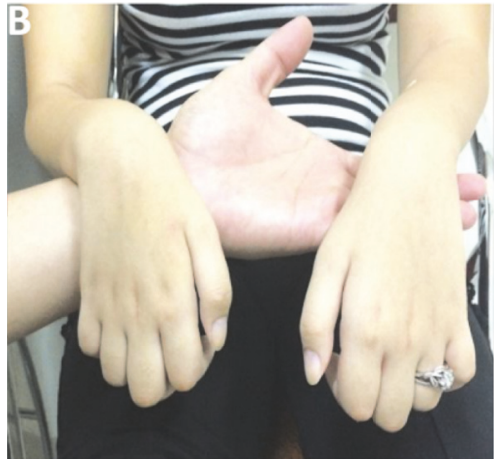

(b)

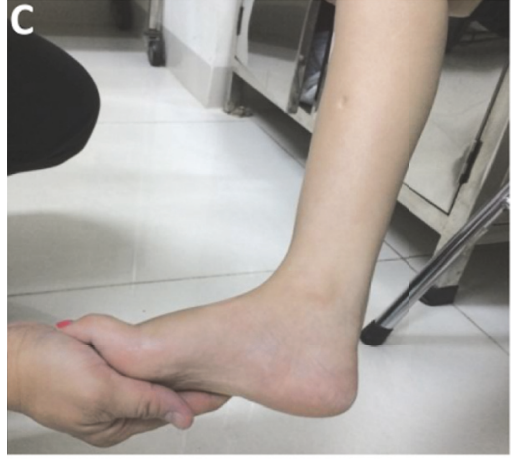

(c)

FIgURE 1: Characteristics of the proband. (a, b) Muscular atrophy in hands; (c) atrophy in the lower legs, pes cavus.

aspects of mitochondrial morphology and functioning, such as on the maintenance and regulation of normal functioning, structural integrity, and intracellular networking of the mitochondria [6]. GDAP1 is identified as a CMT-causative gene $[7,8]$ and its mutations have been shown to be responsible for an early-onset autosomal recessive demyelinating neuropathy [7], an axonal recessive form [8, 9], an intermediate recessive form [10], and a late-onset autosomal dominant axonal form [11-13].

GDAP1 autosomal recessive inherited mutations cause a severe, early onset neuropathy often resulting in wheelchairdependency in the second or third decade. Most of these patients develop unilateral or bilateral vocal cord paresis and diaphragmatic weakness in the latter stages of the disease [14]. It has been suggested that recessive mutations which cause truncating proteins develop a more severe phenotype, while missense mutations may be associated with a slightly milder progression [15]. Otherwise, autosomal dominant inherited mutations cause a much milder phenotype, characterized by adult onset, predominantly distal involvement, and slow progression $[16,17]$.

We herein report the clinical and electrophysiological findings of affected patients in a Vietnamese family with a severe autosomal recessive axonal sensorimotor neuropathy, due to a duplication of 5 nucleotides in coding region of the GDAP1 gene. This novel variant induces a premature stop codon and may contribute to severe loss of function GDAP1.

\section{Patients and Methods}

We studied a Vietnamese family encompassing all three affected children. The proband and her family underwent clinical and electrodiagnostic evaluations. Genomic DNA was extracted from peripheral blood mainly of the proband and family members using a QIAGEN Blood kit according to the manufacturer's instructions and testing for GDAP1 variants. The 6 exons of GDAP1 were amplified by polymerase chain reaction and analyzed by direct sequencing on an ABI3130XL Genetic Analyzer system (Applied Biosystems, USA). Primers for PCR and sequencing reactions were listed in Supplementary Table 1. Some other common CMT genes
(MPZ, GJB1, MFN2, NEFL, and PMP22) have been analyzed as well.

For the new variant in GDAP1 gene, 50 unrelated healthy Vietnamese control participants were screened. The variant was numbered according to the Human Genome Variation Society (HGVS) nomenclature on the basis of standard reference sequences of mRNA (NM_018972.2) and protein (NP_061845.2).

\section{Results}

3.1. Clinical Features. We described symptoms of the proband and her siblings, ranging in age from 3 to 16 years, and their mean onset age was 27 months.

The proband (II-1) had difficulty running starting at 27 months. She was able to independently climb stairs until age 7 and then required ankle foot orthosis at the same year. Due to progressive distal and proximal leg weakness, she needed crutches from age of 10 years. Clinical examination at the age of 16 years showed atrophy of lower legs, lower arms, intrinsic hand muscles, and areflexia. The proximal extremities muscles showed a moderate paresis. There was severe weakness of the lower arm muscles, in particular of the hand muscles. Sensory impairments were not identical in upper and lower extremities: asymmetrical loss of touch and pain sensations with the upper extremities were intact, and vibration was absent in distal. Pes cavus was seen (Figure 1). There was no evidence of hoarseness, vocal cord paresis, diaphragmatic paralysis, or cognitive impairment.

The 12-year-old sister (II-2) and 3-year-old brother (II-3) of the proband were affected, showing the same symptoms as the proband, although to a lesser extent. Both parents (I-1 and I-2) showed no abnormalities on neurological examination and nerve conduction studies (Table 1).

3.2. Electrophysiological Findings. Nerve conduction studies of the proband showed no CMAP or sensory nerve amplitude potential (SNAP), which suggested axonal neuropathy. EMG showed signs of de- and reinnervation in the right anterior tibial muscle. In the brother of the proband, CMAP reduces significantly and MNCV was in normal range (Table 2). 
TABLE 1: Summary of clinical characteristics in proband and other family members.

\begin{tabular}{|c|c|c|c|c|c|}
\hline & II-1 & II-2 & II-3 & $\mathrm{I}-1$ & $\mathrm{I}-2$ \\
\hline Gender, & Female, & Female, & Male, & Male, & Female \\
\hline age at visit & 16 yos & 12 yos & $40 \mathrm{mos}$ & 46 yos & 45 yos \\
\hline Age of independent working & 13 months & 12 months & 12 months & - & - \\
\hline Age of onset & $27 \mathrm{mos}$ & $27 \mathrm{mos}$ & $27 \mathrm{mos}$ & - & - \\
\hline Muscle weakness & DLL $=$ DUL & DLL $>$ DUL & DLL & No & No \\
\hline Muscle atrophy & $\begin{array}{c}\text { Yes } \\
\text { DLL = DUL }\end{array}$ & $\begin{array}{c}\text { Yes } \\
\text { DLL > DUL }\end{array}$ & No & No & No \\
\hline Tendon reflexes & Absent & Absent & Decrease & Normal & Normal \\
\hline \multicolumn{6}{|l|}{ Sensory loss } \\
\hline (i) Pinprick & Yes & Yes & Yes & Normal & Normal \\
\hline (ii) Vibration & Yes & Yes & Yes & Normal & Normal \\
\hline Deformity & Pes cavus & Pes cavus & No & No & No \\
\hline Respiratory failure & No & No & No & No & No \\
\hline
\end{tabular}

DLL: distal lower limbs; DUL: distal upper limbs.

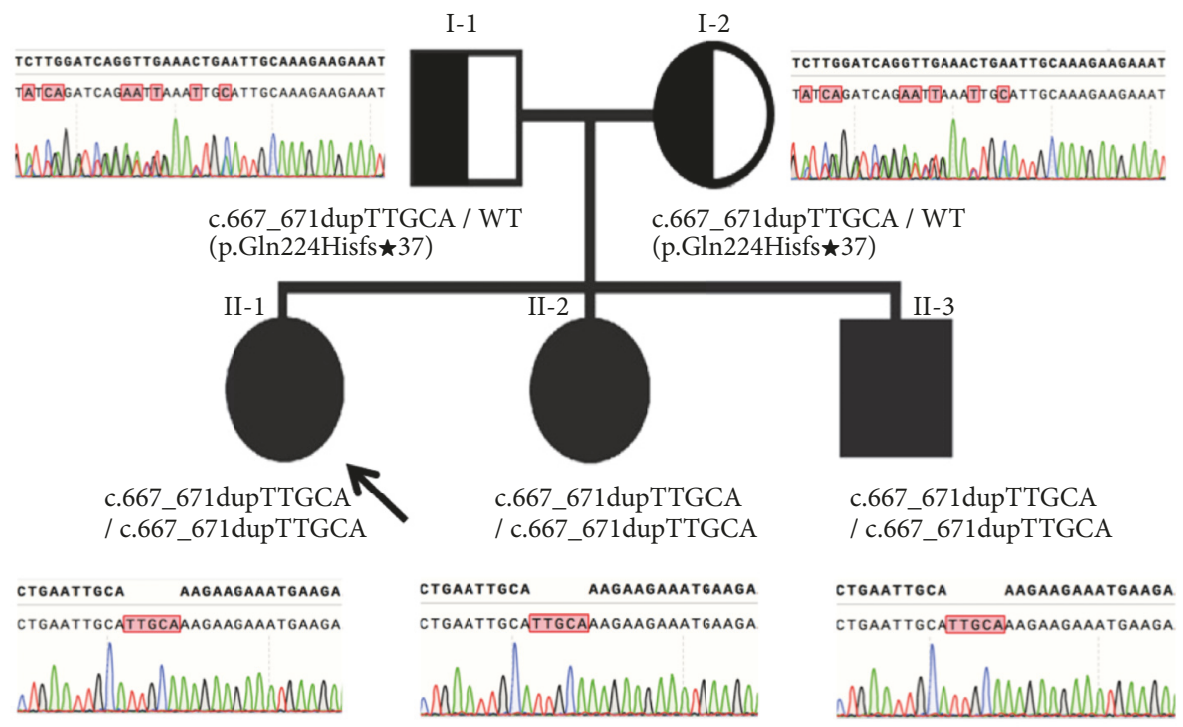

FIGURE 2: Analysis of GDAP1 gene (exon 5) in the unaffected father (I-1), unaffected mother (I-2), the proband (II-1), and her siblings (II-2, II-3).

These data were consistent with a severe sensorimotor axonal polyneuropathy.

3.3. Genetic Analysis. Based on clinical findings, nerve conduction velocities, and family history consistent with autosomal recessive inheritance, a diagnosis of CMT type 4 was suspected. DNA testing revealed a homozygous variation in the GDAP1 gene in the proband and her two siblings: c.667_671dup, and a heterozygosity of the c.667_671dup in both parents (Figure 2). The proband and her two siblings carried a frame shift variant p.Gln224Hisfs $* 37$. However, this alteration was not detected in 50 healthy controls. The Gln224Hisfs $* 37$ is likely pathogenic variant and not previously reported.

\section{Discussion}

This report described a family with axonal CMT where there was a novel GDAP1 variation underlying a typical autosomal recessive phenotype. The affected patients had a disease onset the third year of life with progressive and symmetric hypotrophy and foot deformity. All three of them presented similar disease development and none had mental retardation. The electrodiagnostic results showed a severe axonal neuropathy. It was recorded that the proband's parents are not known to be consanguineous but their origins are from nearby villages in the North area of Vietnam. Both of them have no relevant medical history and no other affected member in multiple generations has been noticed. This inheritance pattern in the pedigree suggests an autosomal recessive disease. 
TABLE 2: Electrodiagnostic findings in proband and other family members.

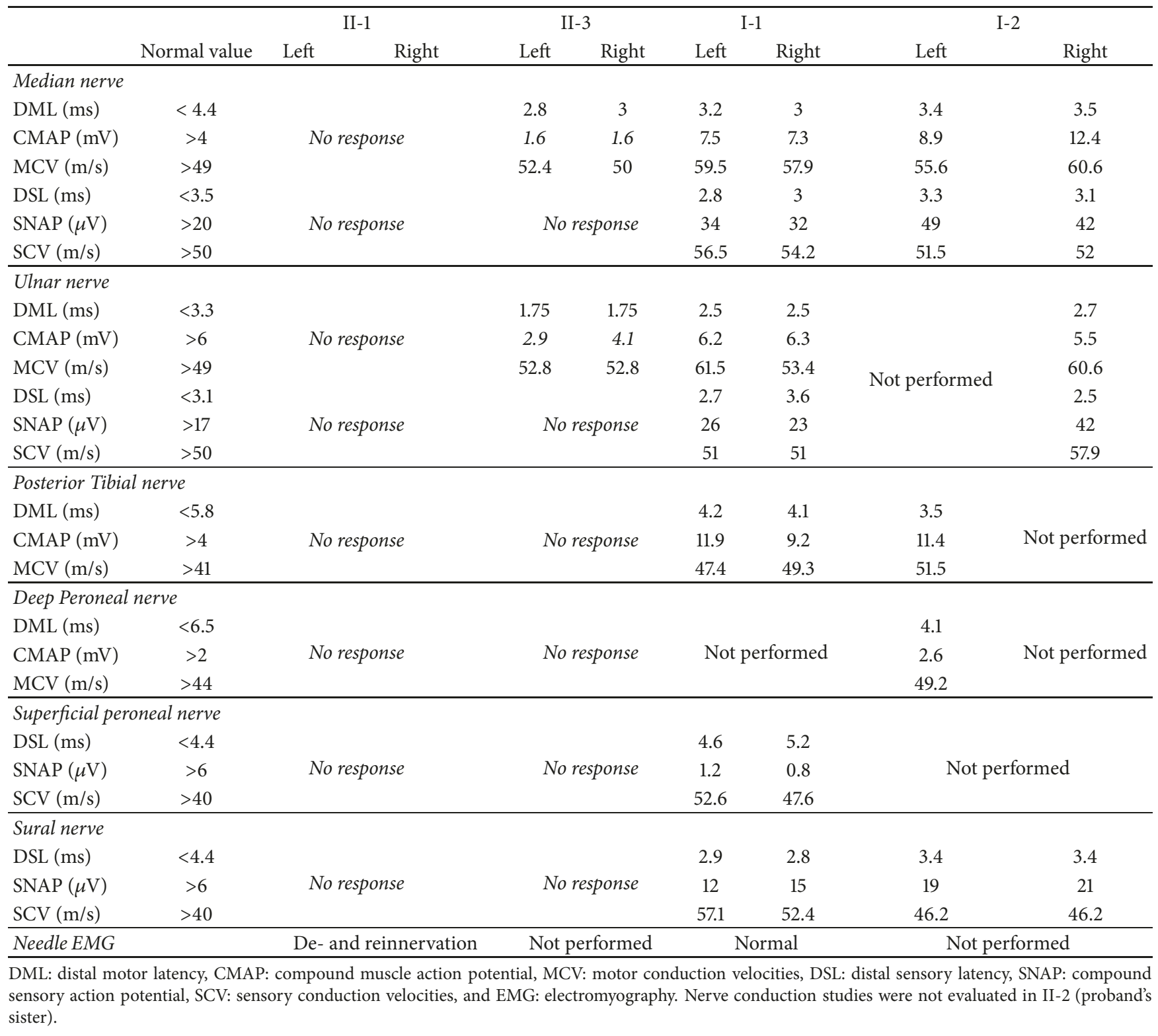

On the basis of a positive familiar history, an axonal sensomotoric neuropathy found in affected patients, early age of onset, and an autosomal recessive pattern of inheritance, alteration of GDAP1 gene was screened in the family. GDAP1 gene sequencing showed a homozygous p.G224Hfs $* 37$ variant, which led us to the confirmation of Charcot-Marie-Tooth disease, type $4 \mathrm{~A}$. Both parents are carriers and without risk of developing the disease. Unfortunately, all three children have the homozygous likely pathogenic variant and present similar disease progression.

GDAP1 belongs to the subfamily of glutathione-Stransferase (GST). It is composed of two typical GST domains (GST-N and GST-C), two alpha helical loops ( $\alpha$-loop), a Cproximal hydrophobic domain (HD1) crucial for GDAP1induced mitochondrial and peroxisomal fission, and a Cterminal transmembrane domain (TMD) essential for the correct locating of the GDAP1 protein [8, 18-22] (Figure 3). To date, more than 80 GDAP1 variants have been implicated in the pathogenesis of CMT. Most are missense and nonsense mutations, and some are frame-shift, deletions, mutations generating truncated and nonfunctional proteins, and those altering the splice sites in the GDAP1 transcripts [15, 23]. Mutations are mostly located in the GST domains of the protein indicating their important roles in the protein function. Most frequent protein consequences are missense followed by frame-shift, nonsense, and splice site mutations. Among the recessively inherited changes, nonsense and frameshift mutations leading to the truncation of the protein produced are very often associated with a most severe phenotype of CMT showing a more rapid course of the disease [15].

GDAP1 mutations are rare in Asian populations, with a reported frequency from $0.6 \%$ to $2.37 \%$ in Japanese and 


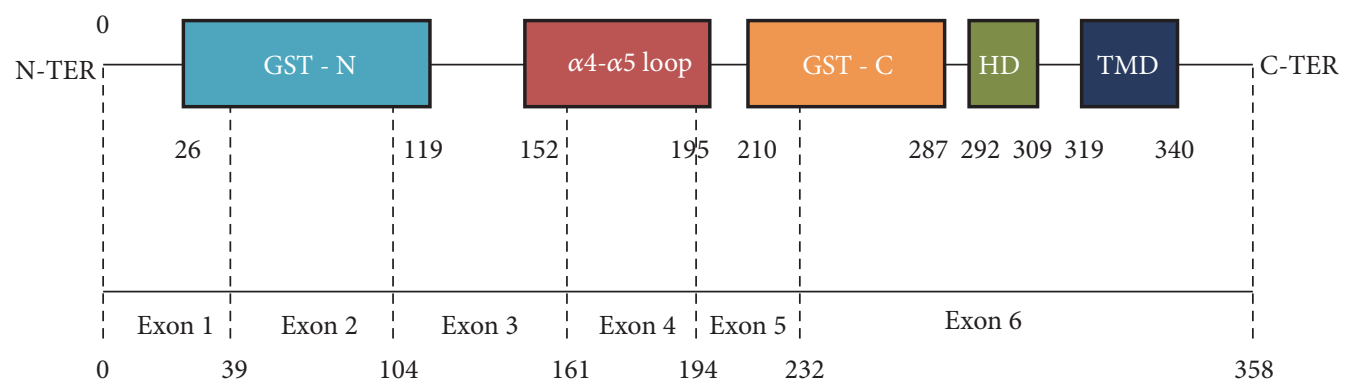

FIGURE 3: Schematic representation of GDAP1. Glutathione-S transferase (GST), hydrophobic domain (HD), transmembrane domain (TMD), $\mathrm{N}$-terminal $(N-T E R)$, and C-terminal (C-TER). The number indicates the amino acid position in GDAP1 protein.

Chinese CMT patients [24-26]. Conversely, high GDAP1 mutation frequencies are observed in European CMT patients, with reported frequencies approximately $7-14 \%$ [27-32].

The p.G224Hfs $* 37$ variant reported in this study has not been described elsewhere. The mutant protein would thus be shortened to the first 223 amino acids instead of 358 (exon 1 to exon 5), followed by an addition of 37 amino acids and a premature stop codon. We consider this variant very likely pathogenic since GST-C domain has important roles in the protein function, and the premature stop codon inducing a truncated protein may impair some involving processes. Furthermore, DNA-testing revealed no mutations in other common CMT genes and no similar variation is detected in healthy control group. This case illustrates the challenges in elucidating the genetic cause in CMT families as GDAP1CMT diseases.

\section{Conclusion}

This work broadens the genetic spectrum of CMT associated with GDAP1 mutations with the identification of new G224Hfs $* 37$ alteration and emphasizes the importance of clinical clues in the diagnosis of inherited neuropathies. It is possible that there are many factors contributing and modulating the GDAP1-CMT and each of the mutations turns the disease in different way, affecting some processes more and some less, thereby explaining such diverse course and severity of CMT disease. More comprehensive information of the genetic background of CMT disease in the Vietnamese is needed in order to describe and understand the effects of mutation on clinical course and prognosis, elucidate the correlation between the genotypes and the clinical phenotypes, and determine the attribution of certain geographical distribution, ethnic background, and areas of high consanguinity.

\section{Data Availability}

The data used to support the findings of this study are included within the article.

\section{Ethical Approval}

This study was approved by the Medical Ethnic Board of University of Medicine and Pharmacy at Ho Chi Minh City, Vietnam. The proband and her family members and healthy control volunteers provided written, informed consents to participate.

\section{Conflicts of Interest}

The authors declare that they have no conflicts of interest.

\section{Acknowledgments}

The authors appreciate the participation and cooperation of the patient, her family, and volunteers. This study was partially supported by The University of Medicine and Pharmacy at Ho Chi Minh City.

\section{Supplementary Materials}

Supplementary Table 1: primers for PCR amplification and sequencing of GDAP1 gene. (Supplementary Materials)

\section{References}

[1] V. Fridman, B. Bundy, M. M. Reilly et al., "CMT subtypes and disease burden in patients enrolled in the Inherited Neuropathies Consortium natural history study: a cross-sectional analysis," Journal of Neurology, Neurosurgery \& Psychiatry, vol. 86, no. 8, pp. 873-878, 2015.

[2] L. Gutmann and M. Shy, "Update on charcot-marie-tooth disease," Current Opinion in Neurology, vol. 28, no. 5, pp. 462467, 2015.

[3] V. Timmerman, A. V. Strickland, and S. Züchner, "Genetics of charcot-marie-tooth (CMT) disease within the frame of the human genome project success," Gene, vol. 5, no. 1, pp. 13-32, 2014.

[4] A. E. Harding and P. K. Thomas, "The clinical features of hereditary motor and sensory neuropathy types I and II," Brain, vol. 103, no. 2, pp. 259-280, 1980.

[5] J. M. Wilmshurst and R. Ouvrier, "Hereditary peripheral neuropathies of childhood: An overview for clinicians," Neuromuscular Disorders, vol. 21, no. 11, pp. 763-775, 2011.

[6] A. Niemann, M. Ruegg, V. La Padula, A. Schenone, and U. Suter, "Ganglioside-induced differentiation associated protein 1 is a regulator of the mitochondrial network: New implications for Charcot-Marie-Tooth disease," The Journal of Cell Biology, vol. 170, no. 7, pp. 1067-1078, 2005.

[7] R. V. Baxter, K. B. Othmane, J. M. Rochelle et al., "Gangliosideinduced differentiation-associated protein-1 is mutant in 
Charcot-Marie-Tooth disease type 4A/8q21," Nature Genetics, vol. 30, no. 1, pp. 21-22, 2002.

[8] A. Cuesta, L. Pedrola, T. Sevilla et al., "The gene encoding ganglioside-induced differentiation-associated protein 1 is mutated in axonal Charcot-Marie-Tooth type 4A disease," Nature Genetics, vol. 30, no. 1, pp. 22-25, 2002.

[9] A. Bouhouche, N. Birouk, A. Benomar, R. Ouazzani, T. Chkili, and M. Yahyaoui, "A novel GDAP1 mutation P78L responsible for CMT4A disease in three Moroccan families," Canadian Journal of Neurological Sciences, vol. 34, no. 4, pp. 421-426, 2007.

[10] J. Senderek, C. Bergmann, V. T. Ramaekers et al., "Mutations in the ganglioside-induced differentiation-associated protein1 (GDAP1) gene in intermediate type autosomal recessive Charcot-Marie-Tooth neuropathy," Brain, vol. 126, no. 3, pp. 642-649, 2003.

[11] C. Casasnovas Pons, I. Banchs, J. Cassereau et al., "Phenotypic spectrum of MFN2 mutations in the Spanish population," Journal of Medical Genetics, vol. 47, no. 4, pp. 249-256, 2010.

[12] B.-O. Choi, M. S. Lee, S. H. Shin et al., "Mutational analysis of PMP22, MPZ, GJB1, EGR2 and NEFL in Korean CharcotMarie-Tooth neuropathy patients," Human Mutation, vol. 24, no. 2, pp. 185-186, 2004.

[13] L. Pedrola, A. Espert, X. Wu, R. Claramunt, M. E. Shy, and F. Palau, "GDAP1, the protein causing Charcot-Marie-Tooth disease type 4A, is expressed in neurons and is associated with mitochondria," Human Molecular Genetics, vol. 14, no. 8, pp. 1087-1094, 2005.

[14] T. Sevilla, T. Jaijo, D. Nauffal et al., "Vocal cord paresis and diaphragmatic dysfunction are severe and frequent symptoms of GDAP1-associated neuropathy," Brain, vol. 131, no. 11, pp. 3051-3061, 2008.

[15] J. Cassereau, A. Chevrollier, N. Gueguen et al., "Mitochondrial dysfunction and pathophysiology of Charcot-Marie-Tooth disease involving GDAP1 mutations," Experimental Neurology, vol. 227, no. 1, pp. 31-41, 2011.

[16] R. Sivera, C. Espinõs, J. J. Vílchez et al., "Phenotypical features of the p.R120W mutation in the GDAP1 gene causing autosomal dominant Charcot-Marie-Tooth disease," Journal of the Peripheral Nervous System, vol. 15, no. 4, pp. 334-344, 2010.

[17] M. Zimoń, J. Baets, G. M. Fabrizi et al., "Dominant GDAP1 mutations cause predominantly mild CMT phenotypes," Neurology, vol. 77, no. 6, pp. 540-548, 2011.

[18] N. Huber, C. Bieniossek, K. M. Wagner et al., "Glutathioneconjugating and membrane-remodeling activity of GDAP1 relies on amphipathic C-terminal domain," Scientific Reports, vol. 6, Article ID 36930, 2016.

[19] N. Huber, S. Guimaraes, M. Schrader, U. Suter, and A. Niemann, "Charcot-Marie-Tooth disease-associated mutants of GDAP1 dissociate its roles in peroxisomal and mitochondrial fission," EMBO Reports, vol. 14, no. 6, pp. 545-552, 2013.

[20] A. Marco, A. Cuesta, L. Pedrola, F. Palau, and I. Marín, "Evolutionary and structural analyses of GDAP1, involved in charcotmarie-tooth disease, characterize a novel class of glutathione transferase-related genes," Molecular Biology and Evolution, vol. 21, no. 1, pp. 176-187, 2004.

[21] A. Niemann, K. M. Wagner, M. Ruegg, and U. Suter, “GDAP1 mutations differ in their effects on mitochondrial dynamics and apoptosis depending on the mode of inheritance," Neurobiology of Disease, vol. 36, no. 3, pp. 509-520, 2009.

[22] K. M. Wagner, M. Rüegg, A. Niemann, and U. Suter, “Targeting and function of the mitochondrial fission factor GDAP1 are dependent on its tail-anchor," PLoS ONE, vol. 4, no. 4, Article ID e5160, 2009.

[23] J. Cassereau, A. Chevrollier, D. Bonneau et al., "A locus-specific database for mutations in GDAP1 allows analysis of genotypephenotype correlations in Charcot-Marie-Tooth diseases type 4A and 2K," Orphanet Journal of Rare Diseases, vol. 6, no. 1, p. 87, 2011.

[24] J. Fu, S. Dai, Y. Lu et al., "Similar clinical, pathological, and genetic features in Chinese patients with autosomal recessive and dominant Charcot-Marie-Tooth disease type 2K," Neuromuscular Disorders, vol. 27, no. 8, pp. 760-765, 2017.

[25] K.-P. Lin, B.-W. Soong, C.-C. Yang et al., "The mutational spectrum in a cohort of charcot-marie-tooth disease type 2 among the han chinese in Taiwan," PLoS ONE, vol. 6, no. 12, Article ID e29393, 2011.

[26] A. Yoshimura, J.-H. Yuan, A. Hashiguchi et al., "Clinical and mutational spectrum of Japanese patients with Charcot-MarieTooth disease caused by GDAP1 variants," Clinical Genetics, vol. 92, no. 3, pp. 274-280, 2017.

[27] M. Auranen, E. Ylikallio, J. Toppila, M. Somer, S. Kiuru-Enari, and H. Tyynismaa, "Dominant GDAP1 founder mutation is a common cause of axonal Charcot-Marie-Tooth disease in Finland," neurogenetics, vol. 14, no. 2, pp. 123-132, 2013.

[28] C. Crimella, A. Tonelli, G. Airoldi et al., "The GST domain of GDAP1 is a frequent target of mutations in the dominant form of axonal Charcot Marie Tooth type 2K," Journal of Medical Genetics, vol. 47, no. 10, pp. 712-716, 2010.

[29] F. Manganelli, S. Tozza, C. Pisciotta et al., "Charcot-MarieTooth disease: Frequency of genetic subtypes in a Southern Italy population," Journal of the Peripheral Nervous System, vol. 19, no. 4, pp. 292-298, 2014.

[30] I. Pezzini, A. Geroldi, S. Capponi et al., "GDAP1 mutations in Italian axonal Charcot-Marie-Tooth patients: Phenotypic features and clinical course," Neuromuscular Disorders, vol. 26, no. 1, pp. 26-32, 2016.

[31] R. Sivera, T. Sevilla, J. J. Vílchez et al., "Charcot-Marie-Tooth disease: genetic and clinical spectrum in a Spanish clinical series," Neurology, vol. 81, no. 18, pp. 1617-1625, 2013.

[32] M. Zimoń, E. Battaloğlu, Y. Parman et al., "Unraveling the genetic landscape of autosomal recessive Charcot-Marie-Tooth neuropathies using a homozygosity mapping approach," neurogenetics, vol. 16, no. 1, pp. 33-42, 2015. 


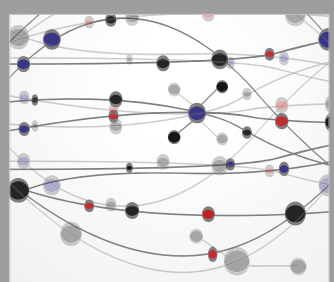

The Scientific World Journal
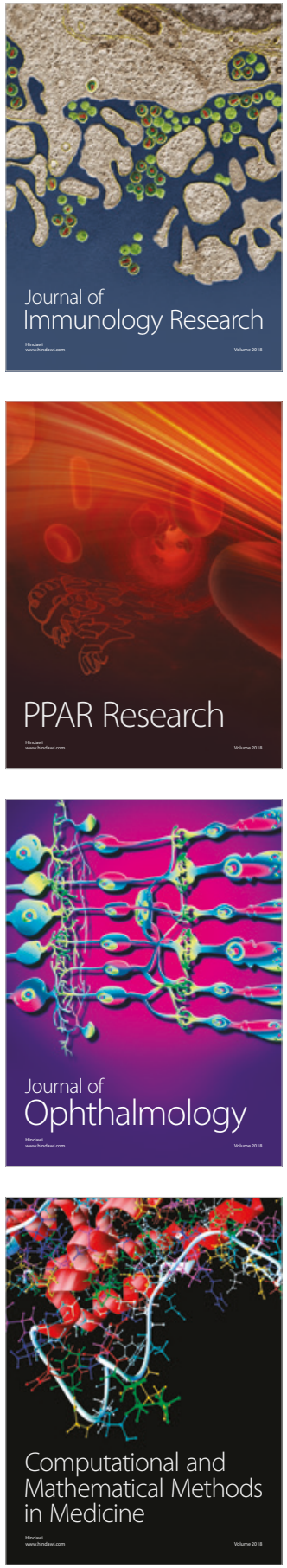

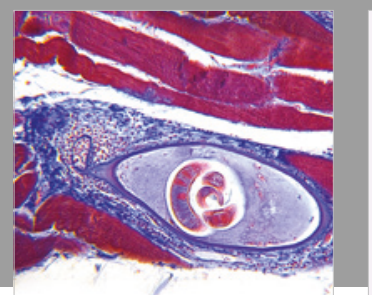

Gastroenterology Research and Practice

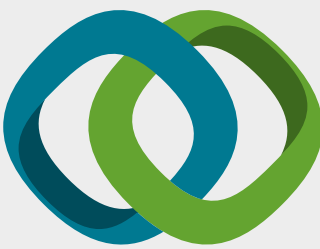

\section{Hindawi}

Submit your manuscripts at

www.hindawi.com
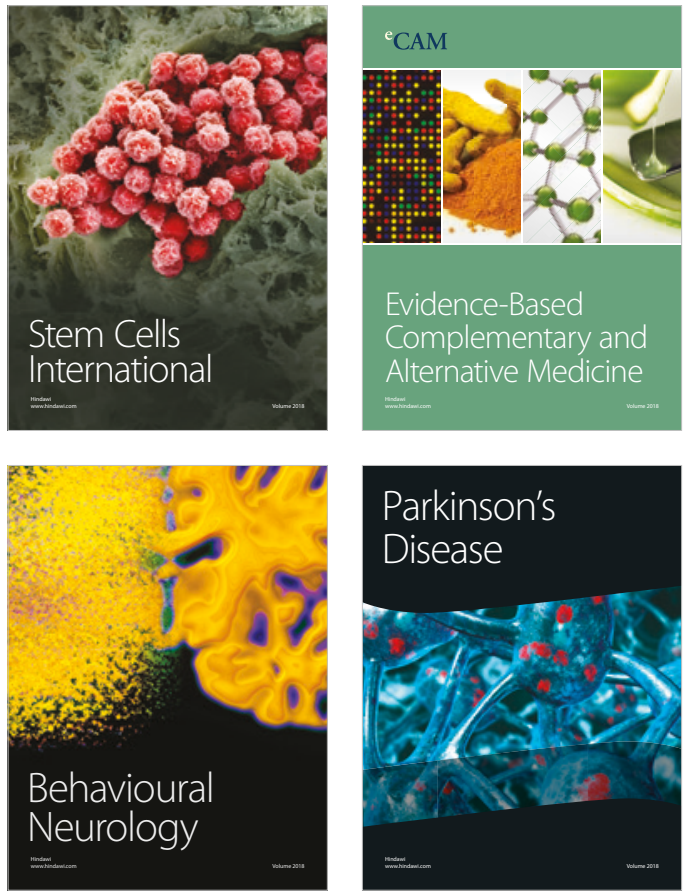

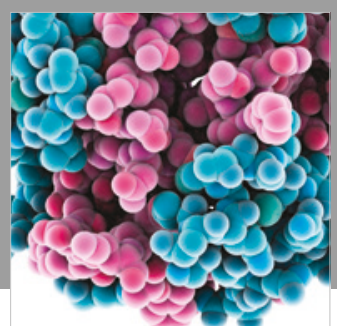

ournal of

Diabetes Research

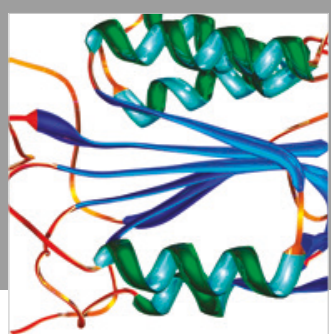

Disease Markers
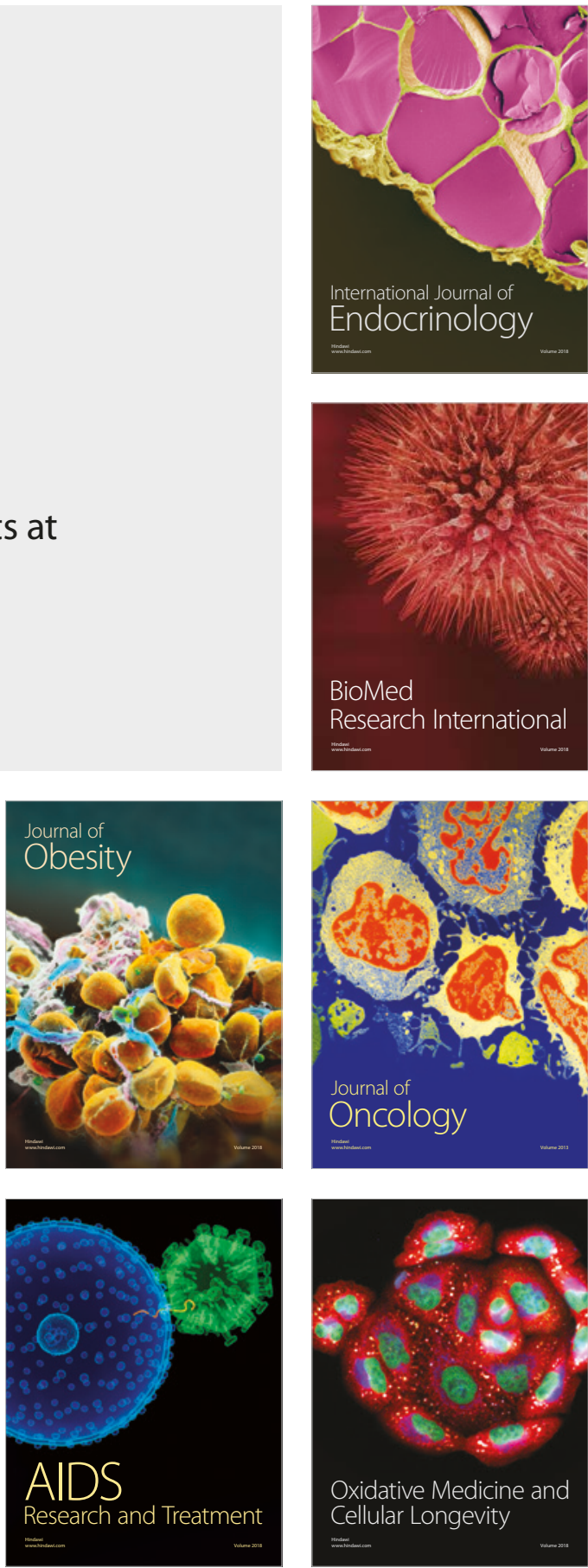